\title{
Effects of Playful Exercise of Older Adults on Balance and Physical Activity: a Randomized Controlled Trial
}

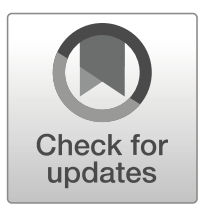

\author{
Humira Ehrari ${ }^{1} \cdot$ Rasmus Tolstrup Larsen ${ }^{2} \cdot$ Henning Langberg ${ }^{3}$ • \\ Henning Boje Andersen ${ }^{1}$ (D)
}

Received: 24 October 2019 / Accepted: 4 February 2020 / Published online: 18 February 2020

(C) The Author(s) 2020

\begin{abstract}
There is evidence that one of the most important approaches to improving the healthy ageing of older adults is for them to carry out daily physical activity. However, motivation to engage in physical activity is often low in old age. This study investigated the potential of engaging older adults in playful exercise to increase physical activity and balance. A randomised control trial (RCT) was performed with 26 independently living older adults (initially 38, but 12 were lost to illness or death during the course of the project), mean age 83.54 (SD: 7.12), 19 women. Participants were randomly allocated to intervention $(n=16)$ or control $(n=12)$ (originally 19 in each group). The intervention consisted of playful exercise on Moto tiles $6 * 2$ min twice a week over 10 weeks, while control group participants engaged in normal daily activities.

The intervention group participants improved functional balance (Berg's Balance Score) by an average of 5.02 points, and the control group by 2.58 points $(p=0.11)$. No between-group difference was observed in physical activities outside exercise sessions $(p=0.82)$. The difference in gain of balance as measured by BBS was below statistical significance, as a result of the sample size being too small. However, trial results suggest that older pre-frail and frail adults who engage in a moderate playful exercise programme over at least 10 weeks may potentially experience a modest gain in balance. Moreover, the playful exercise created a joyous social atmosphere among the participants who spontaneously remarked that the play sessions were much more fun than their standard light exercise programme of one hour twice a week. This motivational outcome is important for adherence to any exercise programme and indeed for general well-being.
\end{abstract}

Keywords Gaming · Physical activity Functional ability $\cdot$ Playful exercise $\cdot$ Behaviour change $\cdot$ Postural control

Henning Boje Andersen

hebq@dtu.dk

Extended author information available on the last page of the article 


\section{Introduction}

According to the World Health Organization's report (2009), one area of increasing focus in health is the demographic development and the increasing life expectancy of older adults. The proportion of adults aged 65 or above was estimated at around 521 million in 2011 and this number will grow by 939 million by 2030 (United Nations, 2015) The increased life expectancy imposes age-related challenges, which affect daily life activities and normal functioning. This can cause inactivity and place old people at an increased risk of a variety of chronic diseases and disorders (World Health Organization 2015). These challenges also impose a heavy burden on society in terms of social welfare, healthcare need and the high cost associated with these challenges (Merom et al. 2012). Age-related impairment leads to deterioration of various parameters of physiological aspects important for balance, such as vision, vestibular and proprioceptive senses, and muscle strength. In addition, fall accidents are considered to be one of the most commonplace problems associated with getting older (Pua et al. 2017; Franceschi et al. 2018).

Prior studies suggest that exercise can increase muscle strength, balance, activity of daily living function and walking speed (Yardley et al. 2006; Janssen and LeBlanc 2010; Gillespie et al. 2012; Lee et al. 2012).

Especially for old people, physical activity can help maintain a high functional capacity, (i.e., the ability to cope with everyday life). For example, exercise interventions among older adults can reduce falls (Sherrington et al. 2011; Gillespie et al. 2012; Guirguis-Blake et al. 2018). Physical activity can prevent the development of lifestyle diseases (e.g., type 2 diabetes and cardiovascular disease) (Reiner et al. 2013; Peek et al. 2016; Daskalopoulou et al. 2017). It can simultaneously reduce symptoms and be included in the treatment of many serious diseases (Blair and Brodney 2018). Finally, physical activity contributes to good mental health, including enhanced self-confidence and joy of life, better social well-being and more energy (Newson and Kemps 2006; Yates et al. 2008; Bauman et al. 2016).

In addition, important dimensions of physical activity include muscle strength and balance training, which also have a major role in health promotion and disease prevention in older adults (Garber et al. 2011).

The physical activity covers all forms of movement that increase energy consumption (e.g., from sports and exercise, to everyday activities such as gardening, cycling, taking the stairs or walking the dog (Titze and Marti 1997)).

Despite documentation of the physical and psychological benefits derived from physical activity, studies also conclude that older adults lack the motivation to get regularly physical active (Phillips et al. 2004; Schutzer and Graves 2004). Therefore, effective strategies to prevent loss of muscle strength, and maintain balance in older adults are needed.

In the present Randomised Controlled Trial we aim to add to the underexplored field of playful exercise for health research by examining how and to what extent playful exercise may empower older adults' functional and physical ability. Hence, we examine to what extent playful physical exercise during a 12-week period improves physical and functional abilities and to what extent it is accompanied by changes in physical activities outside exercise sessions. 


\section{Function Ability}

Balance is a central function in most activities of daily living (ADL) (Kalron and Achiron 2013). The reduced balance increases the risk of falling (Pua et al. 2017). As age increases, gradual deterioration of various sensory systems contributes to a worse postural control (Kalron and Achiron 2013).

The balance problems can occur when only one of the different parameters in postural control is weakened because the systems function in a mutual interaction (Pollock et al. 2000; Laughton et al. 2003). In 2010, the World Health Organization released a report with recommendations for preventing falls and thus fractures and other injuries among old people (World Health Organization 2010). The report indicates that preventive measures are an important element in eliminating the problem of fall accidents as they can slow down the number of hospitalisations related to fall accidents, which are otherwise expected to increase, by 2040 , due to the increasing number of people aged above 65 (Daskalopoulou et al. 2017).

In addition, prior studies show a positive association between physical activity and healthy ageing (Schutzer and Graves 2004; World Health Organization 2010; Sun et al. 2013; Bauman et al. 2016; Daskalopoulou et al. 2017).

It is also documented that through targeted intervention, a significant reduction in the risk of falls for this group of elderly people can be achieved (Howe et al. 2007; Tak et al. 2013). This intervention, for example, may consist of medicine optimisation, and physical exercise such as balance strength and walking training (Bauman et al. 2016). Many studies have shown that appropriate exercise can modify and significantly delay the primary risk factors for falls, including poor balance and muscle weakness in old people (Schreiber et al. 1999; Tárraga et al. 2006; Padala et al. 2012; Bauman et al. 2016; Dietlein et al. 2018). They also showed improvement in cognitive and physical function when playing serious games (Larsen et al. 2013). Furthermore, balance training is considered to be one of the most important factors in reducing falls among older adults (Sherrington et al. 2008; Jessen and Lund 2017). In addition, a growing number of studies show that gaming has positive impact on physical functions (Duque et al. 2013; Cho and Lee 2014).

\section{Playful Exercise}

Gamification refers to playful experiences that aim to motivate individuals in performing certain tasks by making them feel in control and aware of their abilities (Jessen 2016). Gamification is mostly used for behaviour change purposes (Cugelman 2013; Larsen et al. 2013; Edwards et al. 2016; Johnson et al. 2016; Fleming et al. 2017), not least for older adults to become more physically active (Barden et al. 2013; Larsen et al. 2013; Sailer et al. 2014; Boot et al. 2016; Skjæret et al. 2016; Skjæret-Maroni et al. 2016; Kappen et al. 2018). Previous studies show that gamification of exercise engages participants and may lead to behavioural change in the short term (Larsen et al. 2013; Jessen and Jessen 2014; Edwards et al. 2016; Skjæret-Maroni et al. 2016; Lee et al. 2018). But it is well known that the motivation to engage in such physical activity becomes reduced when people get older whether it is playful or not. Still, playful activity 
programmes for older adults may have beneficial effects: reported results include improvement in well-being and balance, and lead to social and emotional benefits, (e.g., social interaction, increased self-esteem, positive emotions) (McLaughlin et al. 2012; Larsen et al. 2013; Boot et al. 2016; Edwards et al. 2016; Kaufman et al. 2016; Dietlein et al. 2018).

\section{Methods}

The study design was based on the CONSORT guideline (Chan et al. 2014) and was planned as an RCT with blinded assessment of end points. The intervention group engaged in 12 min of playful exercise (spread over one hour in 2-min minisessions) twice weekly over a planned duration of 12 weeks. The training protocol included a combination of games that train both static and dynamic postural control as well as agility, reaction time, and for most older adults, endurance. Participants in both groups engaged in their normal physical and social activities including twice-weekly joint meals and social activity and one hour's light exercise (mobility training while sitting in chairs). Daily physical activity of both intervention and control group participants was recorded by activity trackers (SENS motion sensors - cf. Figure 1). The device used is a triaxle accelerometer capturing raw triaxle acceleration $( \pm 4 \mathrm{~g})$ at $12.5 \mathrm{~Hz}$. The collected data are wirelessly transferred to a secure cloud server via Bluetooth and can be accessed via a web interface.

The tracker was attached to the leg above the knee with a plaster, changed once a week with assistance from a student. Participants were measured on Berg Balance Score (BBS), 30 Second Chair Stand Test (CST) and 6 Minute Walk Test (6MWT) at the beginning (baseline) of the study, midway, and at the end. After completing the baseline measurements and signing the informed consent, participants were assigned to two groups by block randomisation (Efird 2011).

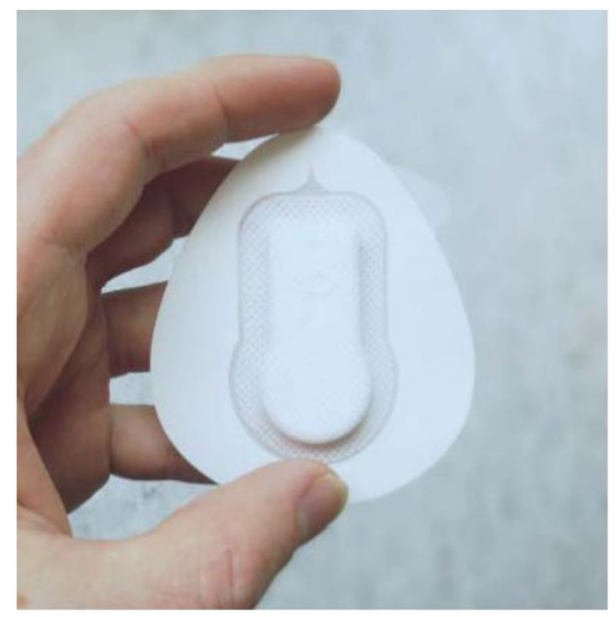

Fig. 1 SENS motion activity tracker 


\section{Randomisation}

For randomisation, an Excel sheet was prepared with the 38 participants' pseudonymised ID-codes, age, gender, and BBS scores. Using Excel's random function repeatedly, the 38 participants were randomly assigned to two groups (intervention and control) calculating for each repetition the group mean age, no. of steps and BBS score and gender distribution. The first repetition of randomised allocation that satisfied the following criterion was chosen: difference of group means less than $<2$ years of age, less than 2 points of BBS, and 4 or more males in each group.

\section{Participants}

The participants were recruited from two activity centres for the elderly in LyngbyTaarbæk municipality and inclusion criteria were: age 65 or above, living independently at home and being able to walk independently and maintain a standing position either alone or with the use of a cane or rollator. Participants signed an informed consent form approved by the Ethics Committee of the technical university of Denmark (DTU DOC 18/00981).

\section{Sample Size}

A sample size calculation was made from literature guidance on (BBS) and results of a previous 12-week feasibility trial with a cohort of elderly 7 participants (March-June 2017; unpublished). The feasibility study showed, as it subsequently became apparent, an untypically high average BBS gain of 11.2 points. The sample size calculation indicated that 2 groups of 20 would be required to detect a BBS gain of at least 5.0 points, using a power estimate of $80 \%$ and a significance threshold of 0.05 .

\section{Materials and Balance Training Programme}

The study used the Moto tiles (www.Moto-tiles.com) as the playful training tool. A Moto tiles set consists of a number of tiles (typically 10) that connect to each other to form a surface. Each tile contains a microprocessor, a battery, IR communication, an FSR sensor, and 8 coloured LEDs in a circle. The FSR sensor can sense a step or a hit on a tile, and the LEDs will shine in different coloured patterns. The tiles communicate with a tablet via Bluetooth. Games are set up via the tablet allowing for selection of any among a variety of different games that challenge the players in ways suitable to their abilities. The Moto tiles are designed to stimulate physical activity level for all ages and train Moto abilities after injuries (Lund 2009; Lund and Marti 2009), and previous studies have reported positive effects on the balance of elderly users (Lund and Jessen 2014; Jessen and Lund 2017). Elderly users typically exercise one by one or in pairs on the tiles during repeated brief game sessions ( $2 \mathrm{~min}$ ), playing interactive games that challenge their dynamic and static balance, agility, endurance, and sensor-Moto reaction. 
The 12-week intervention plan engaged each participant in a training group of 5 elderly users. Each group met at their training centre twice weekly for their 1-h session. Each session was led by two training assistants (students) who managed the individual sessions and supported the elderly in the rare case that they might need physical assistance (Fig. 2). The training protocol included a combination of games that train both static and dynamic postural control. The planned 12-week trial period was divided into two equal periods of 6 weeks, divided by a 3 -week break for Easter holidays. The original plan was to train participants in a training group of 5 elderly people each session, but small adjustments were made from time to time. If participants were prevented from attending their scheduled training session, they were offered a replacement session, sometimes on the same day when they could join the previous or the following team, the session then being extended by 12 min (cf. Fig. 2). Participants in both groups engaged in their normal physical and social activities including their meeting twice weekly at the municipal activity centre for joint meals and social activity and one hour's light exercise (mobility training while sitting in chairs).

\section{Control Group}

Assistants meet with each control group participant once a week for 5-10 min in order to: (a) encourage their continued participation, and (b) check whether activity trackers were functioning and to exchange the plaster holding the tracker.

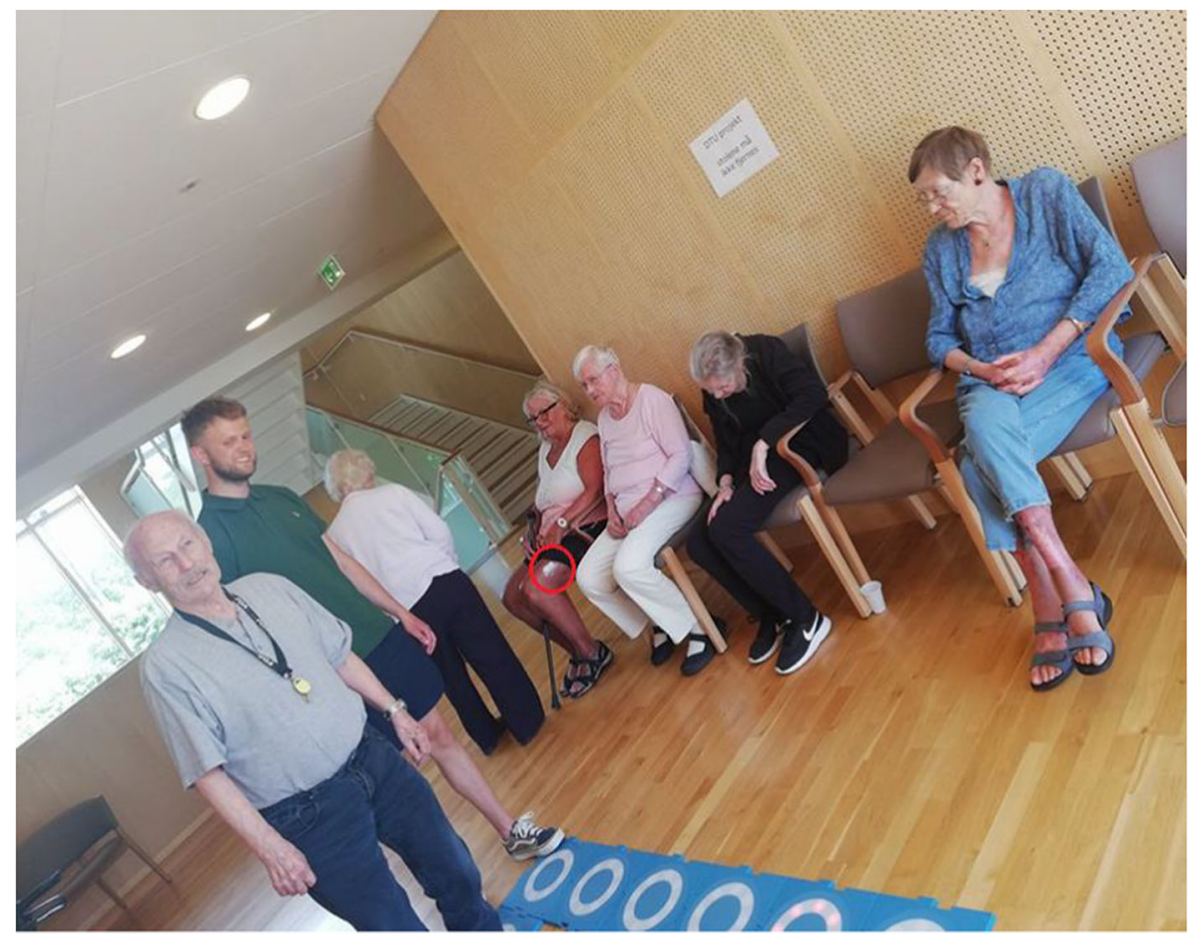

Fig. 2 Training session with Moto tiles (SENS patch encircled in red) 


\section{Primary Outcome}

The primary outcome was balance as measured by BBS. This test is widely used to measure functional balance of old people or others (Rikli and Jones 1999, 2001, 2013; Li 2010). BBS consists of 14 individual tests that reflect movements in everyday life. In each test, the participant receives a score from 0 to 4 depending on their performance. The BBS scores are interpreted as 45-56 = low fall risk, $21-40=$ medium fall risk, 0 $20=$ high fall risk and $<36$ fall risk close to $100 \%$ (Guccione et al. 2012) BBS is widely considered the gold standard for measuring the balance of older users (Langley and Mackintosh 2007).

\section{Secondary Outcomes}

The study had three secondary outcomes: CST, 6MWT and physical activity outside of training:

The CST is used to measure the aerobic capacity of the lower body (Rikli and Jones (2013)). The test is performed in the following steps by instructing the participant: 1 . Sit in the middle of the chair. 2. Place your hands on the opposite shoulder crossed at the wrists. 3. Keep your feet flat on the floor. 4. Keep your back straight, and keep your arms against your chest. 5. On "Go", rise to a full standing position, then sit back down again.

6. Repeat this for $30 \mathrm{~s}$. The number of times the patient comes to a full standing position in $30 \mathrm{~s}$ is then counted. If the patient is over halfway to a standing position when $30 \mathrm{~s}$ have elapsed, it is counted as a stand.

The 6MWT is used to measure aerobic capacity and endurance (Sherwood et al. 2016). The test is performed in these steps: A 20-m course is set up on a hard and level surface. The participants are asked to walk around the course for $6 \mathrm{~min}$. The tester records how many metres are walked. If the participant needs a break this is allowed, while the time keeps going.

Daily physical activity was recorded by SENS motion-sensors 24/7 during the trial period for both intervention and control group participants. Outcome measure was mean number of steps per day.

BBS, CST and 6MWT tests were conducted under the supervision of a certified physiotherapist and three student assistants, all blinded to group allocation.

\section{Ethics}

During recruitment, written and oral information about the study was given to all participants and their relatives. Interested participants were informed that they have the right to withdraw from the trial at any time. Performance data were recorded in a pseudonymous form. All data were collected, transferred and stored in accordance with GDPR guidelines. The study is approved by the Regional Ethics Committee (Center for Regional Udvikling Region Hovedstaden: H-19018499).

\section{Statistical Analysis}

Data were initially examined for normality violations, outliers, errors and missing values. Missing post-trial values were replaced by mid-test values (Telenius et al. 
2015). A two-sample t-test was performed to test between group differences between the pre- and post-trial tests. A $P$ value $<.05$ was considered statistically significant. All statistical analyses were performed with SAS JMP (v.9.4) and Microsoft Excel (2016).

\section{Results}

As shown in Fig. 3, out of 52 possible participants, 38 persons (73\%) met the inclusion criteria and agreed to participate. Two participants $(4 \%)$ have been excluded, not meeting the inclusion criteria, and 12 people $(23 \%)$ dropped out at the very beginning of the trial. During the 12-week study period, 10 out of 38 (26\%) dropped out and $2(5 \%)$ passed away, thus we ended up with data from 26 participants $(14(54 \%)$ in the intervention and 12 persons $(46 \%)$ in the control group) at the end of the trial. The 12 participants who withdrew after randomisation were excluded from the statistical analyses. Final analysis included data from 26 participants (19 females, 7 males; mean age, (S.D) $=83.54$ (7.12) years. All participants skipped one or several sessions due to temporary illness or other obligations. Out of the total of 24 sessions participation median was 20, mean was 18.92 (SD 2.37; max. 23; min. 14) and an attendance rate of $79 \%$. The duration of the exercise is therefore reported for the group as 10 weeks of exercise exposure.

\section{BBS}

Table 1 shows the score on BBS tests at baseline and at the 12-week post-trial. The intervention group increased their score on average by 5.0 points, the control by 2.1 points. The difference between BBS baseline and BBS post-trial for each individual participant in the intervention group ranged from -2 to 11 .

\section{CTS}

The intervention group experienced a decrease in the number of chair stands by 1.00 on average and the control group a decrease of 1.33. The difference was not significance $(p=0.96)$.

\section{MWT}

The intervention group increased their mean 6MWT by 18.5 (SD: 72.2) metres from baseline to post-trial test. The control group increased their 6MWT by 11.0 (SD: 45.7) metres. The difference between the groups was not significant $(p=0.75)$.

\section{Physical Activity}

The control group walked on average 718.53 more daily steps per week (mean = 4859.8; $\mathrm{SD}=356.61$ ) than the intervention group (mean =4140.5; $\mathrm{SD}=418.08)$, $\mathrm{cf}$. Figure 4 . The group difference was not significant ( $p=0.869$; t-test). 


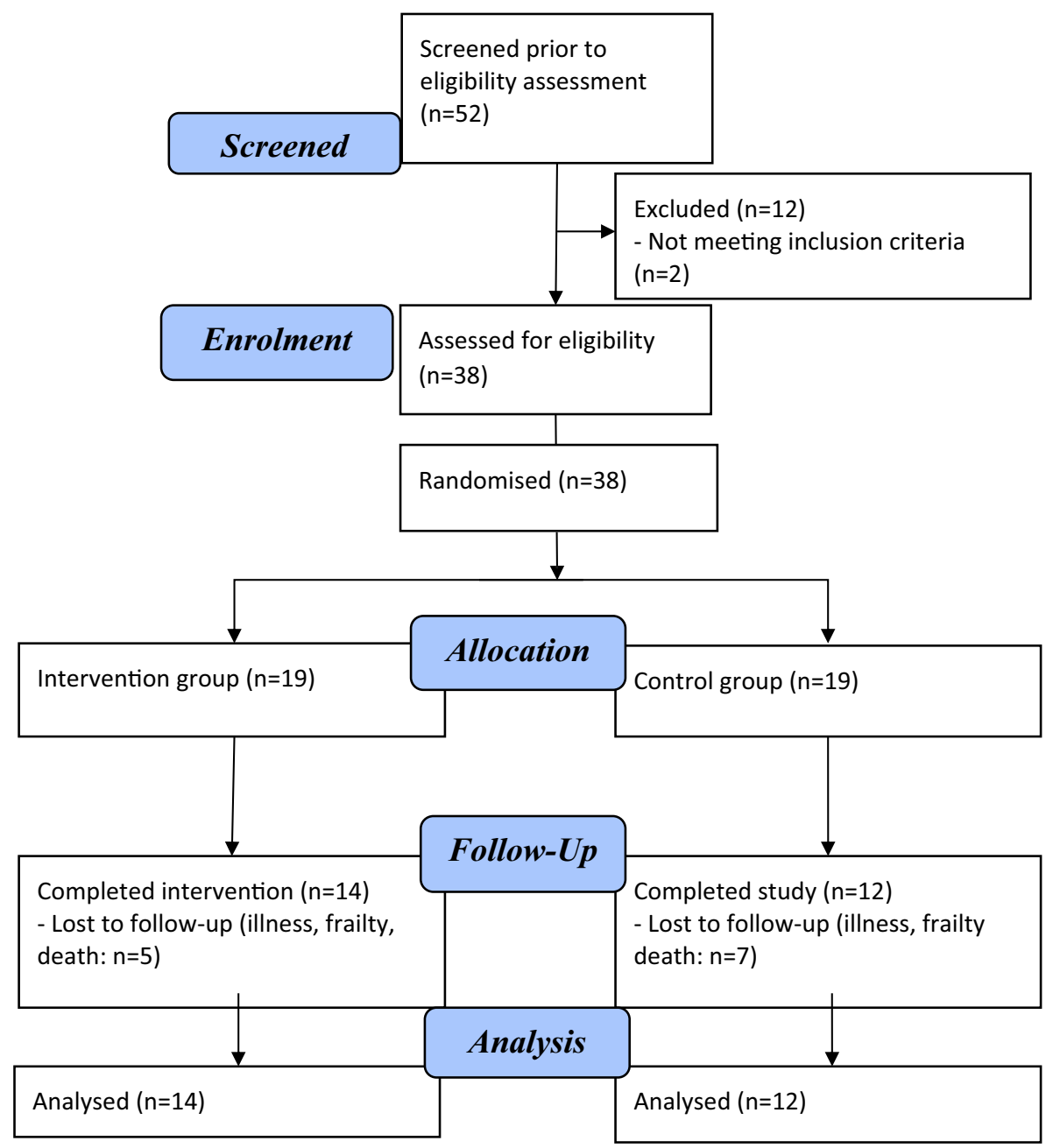

Fig. 3 - Flow chart of the study

\section{Discussion}

This assessor blinded, parallel group, RCT aimed to investigate the effects of a playful exercise intervention with Moto tiles on functional ability and daily physical activity. The study was not sufficiently powered to reveal between-group differences. The mean BBS score of both the control group and the intervention group increased during the 12-week intervention period, but while the observed increments were different between the groups, they did not achieve statistical significance $(p=0.11)$. We have made a post-test power analysis with $80 \%$ power, alpha 0.05 , and with assumptions of a mean difference between intervention and control of 3 points and a pooled SD of 5. This post-test power analysis shows that it would require 45 participants in each group. Still, the randomisation, allocation, assessments and the intervention were found to be applicable and future large-scale RCTs should include sufficient participants to clarify 


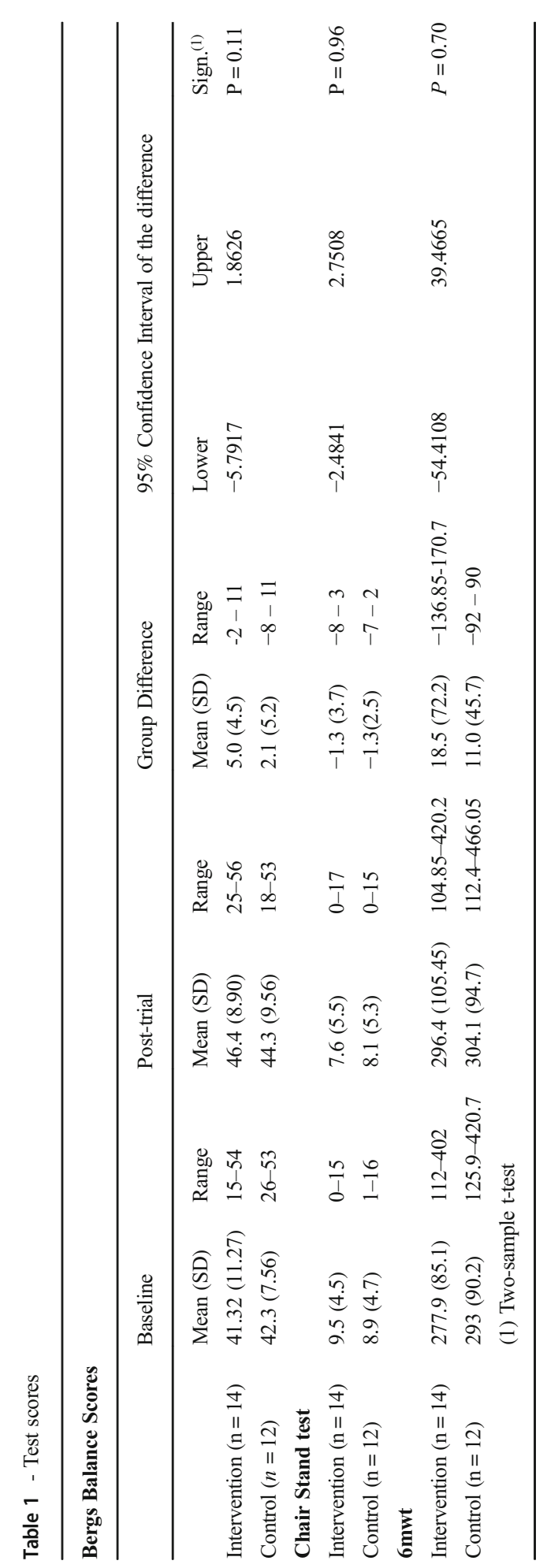




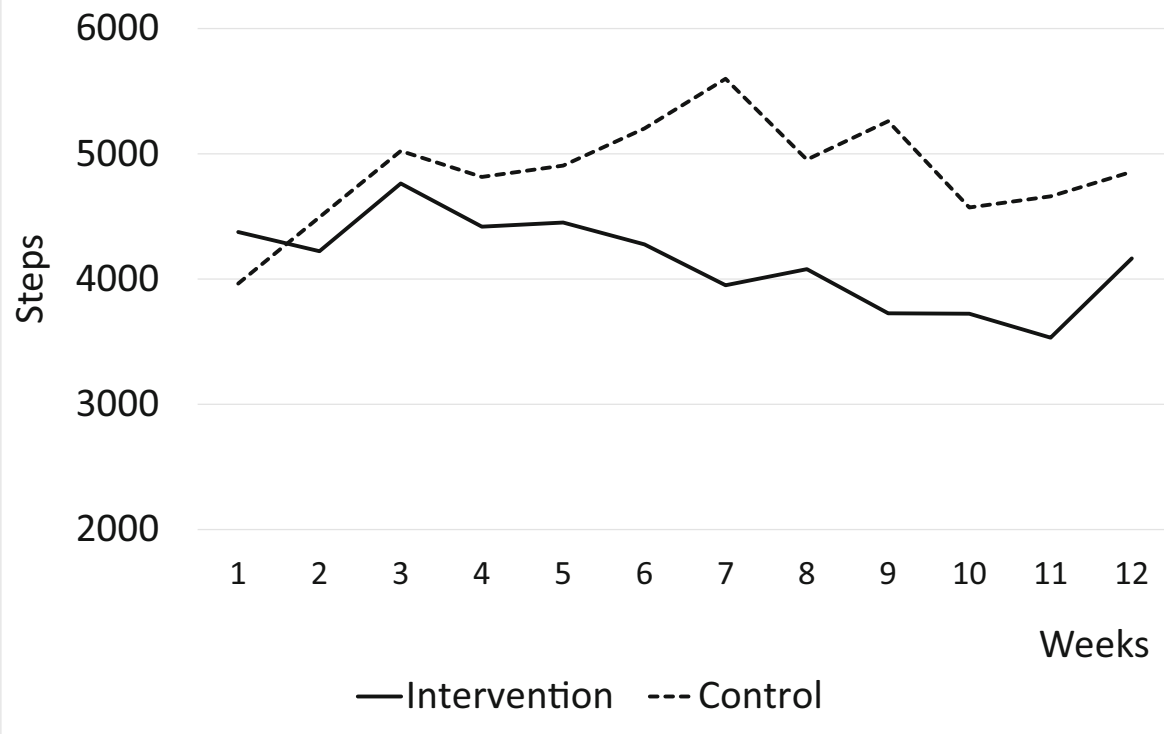

Fig. 4 Weekly mean number of daily steps per group

the effects of the Moto tiles. Moreover, trial results suggest that older pre-frail and frail adults who engage in a moderate playful exercise programme over at least 10 weeks are likely to experience a modest gain in balance.

As the BBS has been found to be with good internal consistency, reliability and good interrater reliability in community-dwelling older adults (Wang et al. 2006; Marques et al. 2016), the measurement properties should have the strength to find clinically relevant differences in such a trial. Furthermore, the BBS has been found to be a valid instrument and is recommended for clinical practice (Marques et al. 2016). Future studies should aim to have sufficient power to investigate clinical relevant differences on the BBS scale which has been reported to be 5 points for a range 35-44 (Donoghue et al. 2009). In this study, the relatively small number $(n=14)$ of participants in the intervention group increased their BBS score by 5 points, which suggests that the intervention period could possibly benefit from being longer in duration or more intense (minutes of playful exercise per week).

Similar conclusions can be drawn from the findings on the CST, the 6MWT and the daily physical activity. No between-group differences were found. As the study was not powered for such an investigation, the null-hypothesis cannot be confirmed and future investigations could reveal relevant clinical differences on the abovementioned outcomes.

Previous investigations have found large and promising effects from a Moto tiles intervention (Lund and Jessen 2013; Jessen and Lund 2014; Lund and Jessen 2014). However, we have not been able to replicate these findings, our results indicating modest, non-significant, improvements of balance outcomes. The difference between 
the results of our study and previous studies might be explained by a difference in participant characteristics affecting the results. Nonetheless, the participant populations were relatively similar in regard to age and functioning. In Lund and Jessen 2013 and Jessen and Lund 2014 the Dynamic Gait Index was used. Future studies should investigate if previous findings (Lund and Jessen 2013; Jessen and Lund 2014) occurred by chance or if Moto tile exercise indeed has a large effect on the balance of older adults.

It was noticeable that the playful exercise created a joyous social atmosphere among the participants and they remarked spontaneously that the playful sessions were much more fun than their standard light "chair training". This motivational outcome is important for adherence to any exercise programme - and indeed for general wellbeing.

\section{Limitations}

This RCT comes with several limitations. First of all, this study was originally designed as a normal superiority RCT, powered to investigate the effects of the Moto tiles intervention. After completing the study in the available time, it was not possible to include sufficient participants and no final conclusion can be made upon the study effects or lack thereof. Studies that fail to reach the pre-specified sample size will be prone to type II errors (failing to reject the null-hypothesis when it should be rejected) but most importantly, the confidence intervals of the effect sizes and thus the mean estimates are wide and comes with a high degree of uncertainty. The conclusion should instead be focused on applicability and adherence to the intervention.

Secondly, and probably most relevant, we did not measure the participants' quality of life, motivation or self-efficacy for exercise. In hindsight, instruments such as the Short Form 36 could have produced useful valid information on the quality of life effects of the intervention (Walters et al. 2001). Similarly, instruments such as the selfefficacy for exercise questionnaire could have been included to clarify the motivation and self-efficacy for exercise and physical activity (Resnick and Jenkins 2000). In summary, to provide the most useful results on the feasibility of the intervention, adherence and motivation for participation should be investigated using specific instruments.

\section{Conclusion}

This study investigated an innovative playful exercise intervention with Moto tiles in a population of older adults. The randomisation process, allocation of participants, 24/7 tracking of physical activity over many weeks, outcome assessments and adherence of both intervention and control participants were found to be applicable. Future large-scale RCTs should include sufficient participants to clarify the effects of the Moto tiles and whether the intervention provides a clinically relevant effect on the functional abilities of older adults. Furthermore, future studies should use specific instruments to investigate the effects on quality 
of life, motivation and self-efficacy for exercise and physical activity in older adults.

Acknowledgments The work presented in this paper is part of project REACH (http:lcrreach2020.eu) that received funding from the European Union's Horizon 2020 Research and Innovation program under grant agreement No 690425. The authors are grateful to our REACH partners for support and to all project contributors: our very dedicated participating citizens of the two care centres, Bredebo and Virumgård, staff at the centres, our student assistants and physiotherapist Mr. Andreas Lund Hessner. We also thank Mr. Christian Bøge Lyndegaard. DTU, for statistical analysis and advice.

\section{Compliance with Ethical Standards}

Competing Interests The authors declare that they have no competing interests.

Open Access This article is licensed under a Creative Commons Attribution 4.0 International License, which permits use, sharing, adaptation, distribution and reproduction in any medium or format, as long as you give appropriate credit to the original author(s) and the source, provide a link to the Creative Commons licence, and indicate if changes were made. The images or other third party material in this article are included in the article's Creative Commons licence, unless indicated otherwise in a credit line to the material. If material is not included in the article's Creative Commons licence and your intended use is not permitted by statutory regulation or exceeds the permitted use, you will need to obtain permission directly from the copyright holder. To view a copy of this licence, visit http://creativecommons.org/licenses/by/4.0/.

\section{References}

Barden, P., Curzon, P. \& McOwan, P. (2013) 'Gameful systems: Play in the digital age for young and old', ACM CHI'13, April 27 - May 2, 2013,.

Bauman, A., et al. (2016). Updating the evidence for physical activity: Summative reviews of the epidemiological evidence, prevalence, and interventions to promote "active aging". Gerontologist. https://oi. org/10.1093/geront/gnw031.

Blair, S., \& Brodney, S. (2018). Effects of physical inactivity and obesity on morbidity and mortality: Current evidence and research issues. Rehabilitation Oncology. https://doi.org/10.1097/01893697-200119020-00028.

Boot, W. R., et al. (2016). 'The gamification of cognitive training: Older adults' perceptions of and attitudes toward digital game-based interventions'. In Lecture Notes in Computer Science (including subseries Lecture Notes in Artificial Intelligence and Lecture Notes in Bioinformatics). https://doi.org/10.1007/9783-319-39943-0 28.

Chan, L., Heinemann, A. W., \& Roberts, J. (2014). Elevating the quality of disability and rehabilitation research: Mandatory use of the reporting guidelines. American Journal of Occupational Therapy. https://doi.org/10.5014/ajot.2014.682004.

Cho, K. H., \& Lee, W. H. (2014). Effect of treadmill training based real-world video recording on balance and gait in chronic stroke patients: A randomized controlled trial. Gait and Posture. https://doi.org/10.1016/j. gaitpost.2013.09.003.

Cugelman, B. (2013). Gamification: What it is and why it matters to digital health behavior change developers. Journal of Medical Internet Research. https://doi.org/10.2196/games.3139.

Daskalopoulou, C., et al. (2017). Physical activity and healthy ageing: A systematic review and meta-analysis of longitudinal cohort studies. Ageing Research Reviews. https://doi.org/10.1016/j.arr.2017.06.003.

Dietlein, C., et al. (2018). Feasibility and effects of serious games for people with dementia: A systematic review and recommendations for future research. Gerontechnology. https://doi.org/10.4017 /gt.2018.17.1.001.00.

Donoghue, D., et al. (2009). How much change is true change? The minimum detectable change of the berg balance scale in elderly people. Journal of Rehabilitation Medicine. https://doi.org/10.2340/16501977-0337.

Duque, G., et al. (2013). Effects of balance training using a virtual-reality system in older fallers. Clinical Interventions in Aging. https://doi.org/10.2147/CIA.S41453.

Edwards, E. A., et al. (2016). Gamification for health promotion: Systematic review of behaviour change techniques in smartphone apps. BMJ Open. https://doi.org/10.1136/bmjopen-2016-012447. 
Efird, J. (2011). Blocked randomization with randomly selected block sizes. International Journal of Environmental Research and Public Health. https://doi.org/10.3390/ijerph8010015.

Fleming, T. M., et al. (2017). Serious games and gamification for mental health: Current status and promising directions. Frontiers in Psychiatry. https://doi.org/10.3389/fpsyt.2016.00215.

Franceschi, C., et al. (2018). The continuum of aging and age-related diseases: Common mechanisms but different rates. Frontiers in Medicine. https://doi.org/10.3389/fmed.2018.00061.

Garber, C. E., et al. (2011). Quantity and quality of exercise for developing and maintaining cardiorespiratory, musculoskeletal, and neuromotor fitness in apparently healthy adults: Guidance for prescribing exercise. Medicine and Science in Sports and Exercise. https://doi.org/10.1249/MSS.0b013e318213fefb.

Gillespie, L. D., et al. (2012). Interventions for preventing falls in older people living in the community. The Cochrane database of systematic reviews. https://doi.org/10.1002/14651858.CD007146.pub2.

Guccione, A. A. et al. (2012) Balance and falls. Third Edit. Mosby. https://doi.org/10.1016/C2009-0-60243-9.

Guirguis-Blake, J. M., et al. (2018). Interventions to prevent falls in older adults: Updated evidence report and systematic review for the US preventive services task force. JAMA - Journal of the American Medical Association. https://doi.org/10.1001/jama.2017.21962.

Howe, T. E., et al. (2007). Exercise for improving balance in older people. Cochrane Database of Systematic Reviews. https://doi.org/10.1002/14651858.CD004963.pub2.

Janssen, I., \& LeBlanc, A. G. (2010). Systematic review of the health benefits of physical activity and fitness in school-aged children and youth. International Journal of Behavioral Nutrition and Physical Activity. https://doi.org/10.1186/1479-5868-7-40.

Jessen, J. D. (2016). Evaluation and understanding of Playware technology - Trials with playful balance training. Technical University of Denmark. doi. isbn:978-87-92465-61-0.

Jessen, J. D., \& Jessen, C. (2014). What games do: Interaction, design, and actor network theory. In ACHI 2014 - 7th International Conference on Advances in Computer-Human Interactions.

Jessen, J. D., \& Lund, H. H. (2017). Study protocol: Effect of playful training on functional abilities of older adults - a randomized controlled trial. BMC Geriatrics., 17, 1-6. https://oi.org/10.1186/s12877-017-0416-5.

Jessen, J. D., Lund, H. H., \& Jessen, C. (2014). Physical computer games for motivating physical play among elderly. In Gerontechnology. https://doi.org/10.4017/gt.2014.13.02.185.00.

Johnson, D., et al. (2016). Gamification for health and wellbeing: A systematic review of the literature. Internet Interventions. https://doi.org/10.1016/j.invent.2016.10.002.

Kalron, A., \& Achiron, A. (2013). Postural control, falls and fear of falling in people with multiple sclerosis without mobility aids. Journal of the Neurological Sciences. https://doi.org/10.1016/j.jns.2013.09.029.

Kappen, D., Mirza-Babaei, P., \& Nacke, L. (2018). Gamification of older adults' physical activity: An eightweek study. In Proceedings of the 51st Hawaii International Conference on System Sciences. https://doi. org/10.24251/hicss.2018.149.

Kaufman, D., et al. (2016). 'Older Adults' Digital Gameplay'. Simulation \& Gaming. https://doi.org/10.1177 $/ 1046878116645736$.

Langley, F. A., \& Mackintosh, S. F. (2007). Functional balance assessment of older community dwelling adults: A systematic review of the literature. Internet Journal of Allied Health Sciences and Practice.

Larsen, L. H., et al. (2013). The physical effect of Exergames in healthy elderly - a systematic review. Games for Health Journal. https://doi.org/10.1089/g4h.2013.0036.

Laughton, C. A., et al. (2003). Aging, muscle activity, and balance control: Physiologic changes associated with balance impairment. Gait and Posture. https://doi.org/10.1016/S0966-6362(02)00200-X.

Lee, I. M., et al. (2012). Effect of physical inactivity on major non-communicable diseases worldwide: An analysis of burden of disease and life expectancy. The Lancet. https://doi.org/10.1016/S0140-6736(12)61031-9.

Lee, D., et al. (2018). Puzzle walk: A gamified mobile app to increase physical activity in adults with autism spectrum disorder. In 2018 10th International Conference on Virtual Worlds and Games for Serious Applications, VS-Games 2018 - Proceedings. https://doi.org/10.1109/VS-Games.2018.8493439.

Li, S. (2010). The balance scale. Nature., 464. https://doi.org/10.1038/464804a.

Lund, H. H. (2009). Modular robotics for playful physiotherapy. In 2009 IEEE International Conference on Rehabilitation Robotics, ICORR 2009. https://doi.org/10.1109/ICORR.2009.5209517.

Lund, H. H., \& Jessen, J. D. (2013). Effect of playful balancing training - a pilot randomized controlled trial'. In Proceedings of 18th International Symposium on Artificial Life and Robotics. Japan: ALIFE ROBOTICS CO, LTD.

Lund, H. H., \& Jessen, J. D. (2014). Effects of short-term training of community-dwelling elderly with modular interactive tiles. Games for Health Journal. https://doi.org/10.1089/g4h.2014.0028.

Lund, H. H., \& Marti, P. (2009). Designing modular robotic playware. In Proceedings - IEEE International Workshop on Robot and Human Interactive Communication. https://doi.org/10.1109 /ROMAN.2009.5326286. 
Marques, A., et al. (2016). Reliability, validity, and ability to identify fall status of the balance evaluation systems test, mini-balance evaluation systems test, and brief-balance evaluation systems test in older people living in the community. Archives of Physical Medicine and Rehabilitation. https://oi. org/10.1016/j.apmr.2016.07.011.

McLaughlin, A., et al. (2012). Putting fun into video games for older adults. Ergonomics in Design. https://doi. org/10.1177/1064804611435654.

Merom, D., et al. (2012). Prevalence and correlates of participation in fall prevention exercise/physical activity by older adults. Preventive Medicine. https://doi.org/10.1016/j.ypmed.2012.10.001.

Newson, R., \& Kemps, E. (2006). Cardiorespiratory fitness as a predictor of successful cognitive ageing. Journal of Clinical and Experimental Neuropsychology. https://doi.org/10.1080/13803390591004356.

United Nations (2015). World Population Ageing. United Nations, Department of Economic and Social Affairs, Population Division. Doi: ST/ESA/SER.A/390.

Padala, K. P., et al. (2012). Wii-fit for improving gait and balance in an assisted living facility: A pilot study. Journal of Aging Research. https://doi.org/10.1155/2012/597573.

Peek, S. T. M., et al. (2016). Older adults' reasons for using technology while aging in place. Gerontology, 62(2), 226-237. https://doi.org/10.1159/000430949.

Phillips, E. M., Schneider, J. C., \& Mercer, G. R. (2004). Motivating elders to initiate and maintain exercise. Archives of Physical Medicine and Rehabilitation. https://doi.org/10.1016/j.apmr.2004.03.012.

Pollock, A. S., et al. (2000). What is balance? Clinical Rehabilitation. https://doi.org/10.1191/0269215500 cr342oa.

Pua, Y. H., Ong, P. H., Clark, R. A., Matcher, D. B., \& Lim, E. C. W. (2017). Falls efficacy, postural balance, and risk for falls in older adults with falls-related emergency department visits: Prospective cohort study. BMC Geriatrics., 17, 1-7. https://doi.org/10.1186/s12877-017-0682-2.

Reiner, M., et al. (2013). Long-term health benefits of physical activity-a systematic review of longitudinal studies. BMC Public Health. https://doi.org/10.1186/1471-2458-13-813.

Resnick, B., \& Jenkins, L. S. (2000). Testing the reliability and validity of the self-efficacy for exercise scale. Nursing Research. https://doi.org/10.1097/00006199-200005000-00007.

Rikli, R. E., \& Jones, C. J. (1999). Development and validation of a functional fitness test for communityresiding older adults. Journal of Aging and Physical Activity. https://doi.org/10.1123/japa.7.2.129.

Rikli, R., \& Jones, C. (2001). Senior fitness test. Champaign (IL): Human Kinetics.

Rikli, R. E., \& Jones, C. J. (2013). Development and validation of criterion-referenced clinically relevant fitness standards for maintaining physical independence in later years. Gerontologist. https:/doi. org/10.1093/geront/gns071.

Sailer, M., et al. (2014). Psychological perspectives on motivation through gamification. In Interaction Design and Architecture(s).

Schreiber, M., et al. (1999). Potential of an interactive computer-based training in the rehabilitation of dementia: An initial study. Neuropsychological Rehabilitation. https://doi.org/10.1080/713755596.

Schutzer, K. A., \& Graves, B. S. (2004). Barriers and motivations to exercise in older adults. Preventive Medicine, 1056-1061. https://doi.org/10.1016/j.ypmed.2004.04.003.

Sherrington, C., et al. (2008). Effective exercise for the prevention of falls: A systematic review and metaanalysis. Journal of the American Geriatrics Society. https://doi.org/10.1111/j.1532-5415.2008.02014.x.

Sherrington, C., et al. (2011). Exercise to prevent falls in older adults: An updated meta-analysis and best practice recommendations. New South Wales Public Health Bulletin. https://doi.org/10.1071/nb10056.

Sherwood, J., et al. (2016). 6-Minute Walk Test. Medicine \& Science in Sports \& Exercise. https://doi. org/10.1249/01.mss.0000485104.47429.b2.

Skjæret, N., et al. (2016). Exercise and rehabilitation delivered through exergames in older adults: An integrative review of technologies, safety and efficacy. International Journal of Medical Informatics. https://doi.org/10.1016/j.ijmedinf.2015.10.008.

Skjæret-Maroni, N., et al. (2016). Exergaming in older adults: Movement characteristics while playing stepping games. Frontiers in Psychology. https://doi.org/10.3389/fpsyg.2016.00964.

Sun, F., Norman, I. J., \& While, A. E. (2013). Physical activity in older people: A systematic review. BMC Public Health. https://doi.org/10.1186/1471-2458-13-449.

Tak, E., et al. (2013). Prevention of onset and progression of basic ADL disability by physical activity in community dwelling older adults: A meta-analysis. Ageing Research Reviews. https://doi.org/10.1016/j. arr.2012.10.001.

Tárraga, L., et al. (2006). A randomised pilot study to assess the efficacy of an interactive, multimedia tool of cognitive stimulation in Alzheimer's disease. Journal of Neurology, Neurosurgery and Psychiatry. https://oi.org/10.1136/jnnp.2005.086074. 
Telenius, E. W., Engedal, K., \& Bergland, A. (2015). Effect of a high-intensity exercise program on physical function and mental health in nursing home residents with dementia: An assessor blinded randomized controlled trial. PLoS One. https://doi.org/10.1371/journal.pone.0126102.

Titze, S. \& Marti, B. (1997) '[Individually adapted counseling about physical activity in medical practice].', Der Orthopade.

Walters, S. J., Munro, J. F., \& Brazier, J. E. (2001). Using the SF-36 with older adults: A cross-sectional community-based survey. Age and Ageing. https://doi.org/10.1093/ageing/30.4.337.

Wang, C. Y., et al. (2006). Psychometric properties of the berg balance scale in a community-dwelling elderly resident population in Taiwan. Journal of the Formosan Medical Association. https://doi.org/10.1016 /S0929-6646(09)60283-7.

World Health Organization. (2009). Global Health risks: Mortality and burden of disease attributable to selected major risks. Bulletin of the World Health Organization, 87, 646-646. https://doi.org/10.2471 /BLT.09.070565.

World Health Organization. (2010). Global recommendations on physical activity for health. Geneva: World Health Organization. https://doi.org/10.1080/11026480410034349.

World Health Organization. (2015). World report on ageing and health. World Health Organization, 1, 1-246. https://doi.org/10.1007/s13398-014-0173-7.2.

Yardley, L., et al. (2006). Older people's views of falls-prevention interventions in six European countries. Gerontologist. https://doi.org/10.1093/geront/46.5.650.

Yates, L. B., et al. (2008). Exceptional longevity in men: Modifiable factors associated with survival and function to age 90 years. Archives of Internal Medicine. https://doi.org/10.1001/archinternmed.2007.77.

Publisher's Note Springer Nature remains neutral with regard to jurisdictional claims in published maps and institutional affiliations.

\section{Affiliations}

\section{Humira Ehrari ${ }^{1} \cdot$ Rasmus Tolstrup Larsen ${ }^{2} \cdot$ Henning Langberg ${ }^{3} \cdot$ Henning Boje Andersen ${ }^{1}$}

Humira Ehrari

humehra@dtu.dk

Rasmus Tolstrup Larsen

rala@sund.ku.dk

Henning Langberg

henninglangberg@gmail.com

1 Technical University of Denmark, DTU Management Department, Akademivej 358, 2800 Lyngby, Denmark

2 Department of Public Health, Section of Social Medicine, University of Copenhagen, Gothersgade 160, 3. sal, 1123 København K, Denmark

3 Copenhagen Healthtech Cluster, Department of Public Health, Section for Health Services Research, University of Copenhagen, Øster Farimagsgade 5, Postboks 2099, 1014 Copenhagen, Denmark 